\title{
Contingent and noncontingent responding in squirrel monkeys as a joint function of quality of, distance from, and schedule of food reinforcement'
}

\author{
EVALYN F, SEGAL \\ SAN DIEGO STATE COLLEGE 2
}

Ss worked on chain FI VI, chain FI DRL, multiple $S^{\Delta}$ VI or mult $S^{\Delta}$ DRL. In chains, onset of the VI or DRL food link was contingent on right-bar presses in the FI link. In multiples, onset of the food component occurred after a pre-set duration of $S^{\Delta}$, and was not response-contingent. Sucrose or peanut pellets reinforced left-bar presses on the food schedules. Variability among $S$ s was great, but generally, results were: (1) where quality of the reinforcer made a difference, sucrose maintained higher response rates than peanut, whether responding was contingently or noncontingently reinforced; (2) responding in the components distal to food was somewhat more sensitive to quality of the reinforcer than responding in the proximal food components; (3) with food reinforcement rate in VI and DRL equated, there was no significant effect of type of schedule per se on FI responding in chains, but (4) there was a differential effect on $S^{\Delta}$ responding in multiples, with less $S^{\Delta}$ responding on mult $S \Delta$ DRL than mult $S \Delta V I$.

This experiment was motivated by our interest in the determinants of psychogenic polydipsia in rats on dryfood, interval reinforcement schedules (Deadwyler et al, 1965; Segal et al, 1965a,b, $c, d, e, f)$. One explanation of such excessive drinking is that it becomes, adventitiously, the first member of a drinking-bar pressing chain, terminated and reinforced by food. Stein (1964) questioned this interpretation, partly on the basis that his rats drank excessively between bar presses only when the reinforcer was dry food, and not when it was milk. This seemed to him evidence that the drinking was merely thirst-induced, and not adventitiously reinforced. But Stein's data showed that not only did drinking decline under milk; bar pressing also declined, suggesting that milk was a weaker reinforcer. Perhaps, then, drinking disappeared because its controlling reinforcement contingencies were too weak: it was only the distal member of only an adventitious response chain terminating in only a weak reinforcer.

This experiment was done to determine what, in fact, would be the effects of changing the quality of reinforcer on behavior that is distal vs. proximal to food, and contingently vs. adventitiously maintained. The experiment was incorporated into a program of research on operant chaining in squirrel monkeys. Method

Four adult, male, squirrel monkeys, maintained at $80 \%$ of ad lib weight, ran in two identical Lehigh 1417 squirrel monkey chambers, each with two levers, a stimulus lampdirectly over each, a food cup, an ad lib water bottle, a ventilator, a speaker feeding white masking noise, a fluorescent houselight, and a one-way vision screen. The chambers were in sound-resistant shells and were put, together, in a sound-resistant, darkened, experimental room that contained white masking noise. Monkeys 1 and 9 ran at the same time of day, as did monkeys 11 and 12. Monkeys 1 and 11 worked in one chamber on the schedules terminating with DRL food reinforcement, and monkeys 9 and 12 in the other chamber on the schedules terminating with VI reinforcement. Reinforcers were Noyes $45 \mathrm{mg}$ pellets made either of sucrose (S) or a mixture of rat chow and peanut (P). The VI schedule was adjusted to yield an average food reinforcement rate equal to that generated by the performance of the DRL Ss; as DRL performance improved, yielding more frequent food reinforcements, the VI reinforcement rate was increased correspondingly. The VI schedule over all sessions reported here was stable at an average inter-reinforcement interval of about $18 \mathrm{sec}$.

Chain FI 2 min. DRL $10 \mathrm{sec}$, or Chain FI 2 VI $18 \mathrm{sec}$

During the FI link, only the lamp above the right bar was lit, green, and right-bar presses produced the conditions for the food (DRL or VI) link on an FI 2. During the food link, only the lamp above the left bar was lit, red, and left-bar presses produced food on DRL $10 \mathrm{sec}$. or VI $18 \mathrm{sec}$. Only one reinforcement was permitted in each cycle; its arrival recycled the chain to the first, FI link. Fifty cycles of the chain were run daily, in sessions that initially had sucrose as the reinforcer. Thereafter, blocks of sucrose and peanut sessions were run until behavior appeared stable under each.

Mult $S^{\Delta} 2 \mathrm{~min}$. DRL $10 \mathrm{sec}$. or Mult $S^{\Delta} 2$ VI $18 \mathrm{sec}$.

The only change was that right-bar presses were ineffective, and 2-min. $S^{\Delta}$ periods marked by a green light over the right bar automatically intervened between each one-reinforcement period on DRL or VI. Sessions began with an $S^{\Delta}$ period and ended with a food reinforcement. Midway through the first peanut block, sessions were lengthened to 75 cycles.

The shift from chains to corresponding multiples permitted evaluation of the effects of the main variable, sucrose vs. peanut, on contingently-reinforced (FI) vs. adventitiously-reinforced $\left(\mathrm{S}^{\Delta}\right)$ responding distal to food. Use of two-component schedules permitted evaluation of the effects of the main variable on responding that was distal (FI or $\mathrm{S}^{\Delta}$ ) vs. proximal (DRL vs. VI) to food.

\section{Results and Discussion}

Figure 1 shows total daily responses of Ss, from the 14th day of the experiment on. By this point behavior was generally appropriate to the contingencies and the VI schedule was stable.

\section{Proximal contingent responding.}

Monkey 1, working on the DRL, showed stable responding that was only slightly higher under sucrose than peanut. Monkey 11 , also working on DRL, showed stable responding for sucrose, but would not eat peanut pellets even when supplementary rations were withheld for a few days, and ceased responding earlier and earlier in each successive peanut block. It was not run at all in the final peanut block. Monkey 12, working on the VI, showed stable responding that did not alter as a function of sucrose vs. peanut. Monkey 9, whose VI behavior was much more variable, showed, at most, only a very slight preference for sucrose.

Distal contingent responding.

Monkey 1 's FI response rate was slightly higher under sucrose than peanut. Behavioral contrast is evident in the fact that rate fell or rose when a shift in reinforcer first occurred, but stabilized at roughly the same level for both reinforcers. Monkey 11 's responding was ex- 


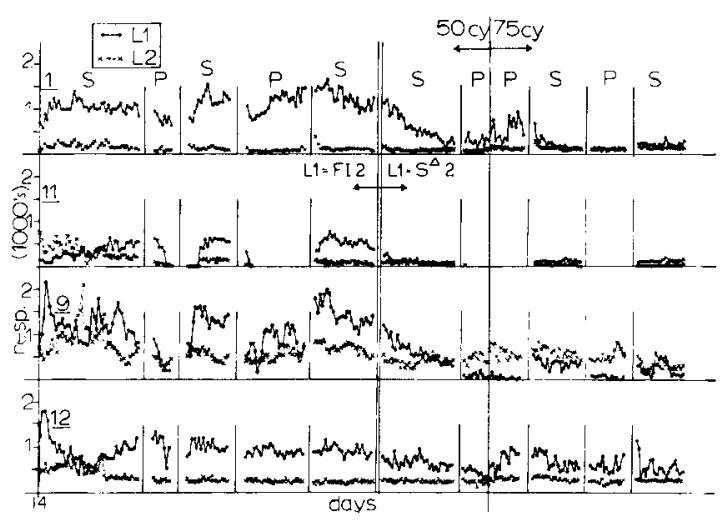

Fig. 1. Total daily responses of each $S$ from day 14 on. Solid lines: right (reinforced) bar presses during the first ( $F I$ or $s \$$ ) "link." Crosses: left (reinforced) bar presses curing the second (VI or DRL) "link." Increases in total daily responses following the shift from 50 to 75 cycles per day reflect the greater length of of sessions, and not increases in response rate.

tremely sensitive to the quality of reinforcer, but it is not possible to compare the effects on proximal and distal responding, in view of the relatively complete absence of responding for peanut. Monkey 9's FI responding was very sensitive to quality of reinforcer, being distinctly higher for sucrose. Monkey 12's FI responding was about as stable as Its VI, and about equally insensitive to the quality of the food.

\section{Summary.}

In those Ss that responded differentially to sucrose vs. peanut $(1,11,9)$, the effect was more marked on distal than proximal responding in monkeys 1 and 9 .

Distal noncontingent responding.

All Ss showed a decline in right-bar pressing when the first component became $S^{\Delta}$, making right-bar presses non-functional. The decline was more rapid, and rates thereafter remained generally lower, in the two DRL monkeys. Monkey 1 showed a very slightly higher $\mathrm{S}^{\Delta}$ response rate in sucrose sessions, evident mainly in the comparison between the last peanut and sucrose blocks. Monkey 11 showed no differential response to sucrose vs. peanut: its $\mathrm{S}^{\Delta}$ responding under sucrose rapidly reached zero, and could not have been lower, and surely would not have been higher, under peanut. Monkey 9 showed a preference for sucrose. Only at the very end of the experiment did it seem that sucrosemaintained $\mathrm{S} \Delta$ responding was extinguishing, although $\mathrm{S}^{\Delta}$ responding under peanut had seemed to be extinguished much earlier. Monkey $12^{\prime}$ 's $\mathrm{S}^{\Delta}$ responding did not decline so far below its FI level as in the other Ss, and the behavior was stable and insensitive to quality of reinforcer, as its FI and VI behavior was.

The fact that the adventitiously-maintained $\mathrm{s}^{\Delta}$ behavior of monkeys 1 and 9 was affected by quality of reinforcer indicates that this variable may, indeed, considerably alter an S's propensity to respond in the distal member of an adventitiously-maintained response chain. Our interpretation of Stein's data on polydipsia as a function of milk vs. pellet reinforcers is at least tenable, and his data do not, of themselves, support the position that polydipsia is entirely thirst-controlled.
First-link responding as a function of the second-link schedule: I. FI.

Except for the aberrant performance of monkey 11, there is no indication in the data of Fig. 1 that making food available on a DRL vs. a VI schedule affected the conditioned reinforcingness of the food-correlated stimuli, as reflected in the FI responding, which was reinforced by onset of those stimuli. Monkeys 1, 9 and 12 all showed comparable $\mathrm{FI}$ rates that varied roughly about $1,000-1,500$ per 50-cycle session. Apparently, the equated food-reinforcement rates yielded about equal conditioned reinforcingness for the correlated stimuli. II. $S \Delta$.

On the other hand, Fig. 1 shows that, overall, there was more $\mathrm{S}^{\Delta}$ responding in VI than in DRL monkeys. Again, the (adventitious) reinforcer for such responding must have been onset of the stimuli correlated with the availability of food, and the FI data indicate that the reinforcement value of these stimuli was about equal in the two groups. The explanation of the differential amount of $\mathrm{S}^{\Delta}$ responding must, then, depend on some other variable. The most likely explanation is induction to $S \Delta$ periods of the response pattern maintained in DRL vs. VI periods. VI maintained a higher response rate than DRL, and this difference apparently generalized to $\mathrm{s} \triangle$ periods.

\section{References}

Deadwyler, S. A., \& Segal, Evalyn F. Determinants of polydipsia: VII. Removing drinking solution midway through DRL sessions. Psychon. Sei.,

Segal, Evalyn F., \& Oden, D. L. Determinants of polydipsia in rats: A reply to Stein. I. Emptying the water bottle, Psychon. Sci., 1965a, 2, 201-202.

Segal, Evalyn F., \& Deadwyler, S. A. Determinants of polydipsia in rats. Il. DRL extinction. Psychon. Sci., 1965b, 2, 203-204.

Segal, Evalyn F., Oden, D. L., \& Deadwyler, S. A. Determinants of polydipsia: III. Withholding food on a free-refnforcement schedule. Psychol. Sci., 1965c, 2, 205-206.

Segal, Evalyn F., Oden, D. L., \& Deadwyler, S, A. Determinants of polydipsia: IV. Free-reinforcement schedules. Psychon. Sci. $1965 \mathrm{~d}, 3,11-12$.

Segal, Evalyn F., Oden, D. L., \& Deadwyler, S. A. Determinants of polydipsia: V. Effect of amphetamine and pentobarbital. Psychon. Sci., 1965e, 3, 33-34.

Segal, Evalyn F., \& Deadwyler, S. A. Determinants of polydipsia: VI. Taste of the drinking solution. Psychon. Sci, 1965f, 3, 101102 .

Stein, L. Excessive drinking in the rat: Superstition or thirst? $J$. comp. physiol. Psychol., 1964, 58, 237-242.

\section{Notes}

1. Supported by NSF GB 1605 and NIMH 8505 .

2. On leave at the Department of Pharmacology, Royal College of Surgeons of England, Lincoln's Inn Fields, London, W.C.2. Send reprint requests to Mr. David L. Oden, Department of Psychology, San Diego State College.

3 . Use of the two different food schedules, with equated reinforcement rates, yielded data on a question extraneous to the main purpurpose of the experiment, but important to the analysis of chaining and conditioned reinforcement, viz., whether the type of primary reinforcement contingency contributes to the conditioned reinforcingness of its correlated stimuli, independently of primary reinforcement rate. 